International Journal of Bifurcation and Chaos

(c) World Scientific Publishing Company

\title{
COMPUTATIONAL INTELLIGENCE METHODS FOR FINANCIAL TIME SERIES MODELING
}

\author{
N.G. PAVLIDIS, D.K. TASOULIS, V.P. PLAGIANAKOS, AND M.N. VRAHATIS \\ Computational Intelligence Laboratory, \\ Department of Mathematics, University of Patras, \\ University of Patras Artificial Intelligence Research Center (UPAIRC), \\ GR-26110 Patras, Greece \\ email: \{npav,dtas,vpp,vrahatis\}@math.upatras.gr
}

\begin{abstract}
In this paper, the combination of unsupervised clustering algorithms with feedforward neural networks in exchange rate time series forecasting is studied. Unsupervised clustering algorithms have the desirable property of deciding on the number of partitions required to accurately segment the input space during the clustering process, thus relieving the user from making this ad hoc choice. Combining this input space partitioning methodology with feedforward neural networks acting as local predictors for each identified cluster helps alleviate the problem of non-stationarity frequently encountered in real-life applications. An improvement in the one-step-ahead forecasting accuracy was achieved compared to a global feedforward neural network model for the time series of the exchange rate of the German Mark to the US Dollar.
\end{abstract}

Keywords: Time Series Modeling and Prediction, Unsupervised Clustering, Neural Networks

\section{Introduction}

System identification and time series prediction are embodiments of the old problem of function approximation [Principe et al., 1998]. A discrete time series is a set of observations of a given variable $z(t)$ ordered according to the parameter time, and denoted as $z_{1}, z_{2}, \ldots, z_{N}$, where $N$ is the size of the time series.

Conventional time series models rely on global approximation, employing techniques such as linear regression, polynomial fitting and artificial neural networks. Global models are well suited to problems with stationary dynamics. In the analysis of real-world systems, however, two of the key problems are non-stationarity (often in the form of switching between regimes) and overfitting (which is particularly serious for noisy processes) [Weigend et al., 1995]. Non-stationarity implies that the statistical properties of the data generator vary through time. This leads to gradual changes in the dependency between the input and output variables.

Noise, on the other hand, refers to the unavailability of complete information from the past behavior of the time series to fully capture the dependency between the future and the past. Noise can be the source of overfitting, which implies that the performance of the forecasting model will be poor when applied to new data [Cao, 
2003; Milidiu \& Renteria, 1999]. Although global approximation methods can be applied to model and forecast time series having the aforementioned characteristics, it is reasonable to expect that the forecasting accuracy can be improved if regions of the input space exhibiting similar dynamics are identified and subsequently a local model is constructed for each of them. A number of researchers have proposed alternative methodologies to perform this task effectively [Cao, 2003; Milidiu \& Renteria, 1999; Pavlidis et al., 2003; Pavlidis et al., 2005; Principe et al., 1998; Sfetsos \& Siriopoulos, 2004; Weigend et al., 1995]. In principal, these methodologies are formed by the combination of two distinct approaches; an algorithm for the partitioning of the input space and a function approximation model. Evidently the partitioning of the input space is critical for the successful application of these methodologies.

In this paper we investigate the improvement in one-step-ahead forecasting accuracy that can be attained if the partitioning of the input space is performed through unsupervised clustering algorithms, while the function approximation model is a feedforward neural network. Clustering can be defined as the process of "grouping a collection of objects into subsets or clusters, such that those within one cluster are more closely related to one another than objects assigned to different clusters" [Hastie et al., 2001]. Unsupervised clustering algorithms automatically approximate the number of clusters in the dataset during their execution. This property is important in the context of partitioning the input space for the purposes of time series forecasting, since the number of partitions corresponding to the different regimes is typically unknown a priori.

As a benchmark we consider the time series of the spot exchange rate of the German Mark against the US Dollar. Foreign exchange rates are among the most important economic indices in international monetary markets. Currently, foreign exchange markets are the most active of all financial markets with average daily trading volumes in traditional (non-electronic broker) estimated at $\$ 1.2$ trillion [Bank of International Settlements, 2001]. Although the precise scale of speculative trading on spot markets is unknown it is estimated that only around $15 \%$ of the trading is driven by non-dealer/financial institution trading. Approximately, $90 \%$ of all foreign currency transactions involve the US Dollar [Bank of International Settlements, 2001]. Foreign exchange rates are affected by many highly correlated economic, political and psychological factors, the interaction of which is in a very complex fashion. Thus, forecasting foreign exchange rates poses many theoretical and experimental challenges [Yao \& Tan, 2000].

The remaining paper is organized as follows: in the next section we present the clustering and neural network algorithms employed in this study. In Section 3 experimental results regarding the spot exchange rate of the German Mark against the US Dollar are presented. The paper ends with a short discussion of the results and concluding remarks. 


\section{Methods}

In this section we briefly describe the application of unsupervised clustering and neural networks in the context of time series modeling and prediction. Furthermore, three unsupervised clustering algorithms, as well as three neural network training algorithms are outlined.

\subsection{Unsupervised Clustering Algorithms}

A critical issue in the process of partitioning the input space for the purpose of time series modeling and forecasting is to obtain an appropriate estimation of the number of subsets. Over- or under-estimation of this quantity can cause the appearance of clusters with little or no physical meaning, and/or clusters containing patterns from regions with different dynamics, and/or clusters with very few patterns that are insufficient for the training of a feedforward neural network.

This is a fundamental and unresolved problem in cluster analysis, independent of the clustering technique applied. For instance, well-known and widely used iterative techniques, such as the Self-Organizing Maps (SOMs) [Kohonen, 1997], the $k$-means algorithm [Hartigan \& Wong, 1979], as well as, the Fuzzy $c$-means algorithm [Bezdek, 1981], require from the user to specify the number of clusters present in the dataset prior to the execution of the algorithm.

On the other hand, algorithms that have the ability to approximate the number of clusters present in a dataset belong to the category of unsupervised clustering algorithms. In this study we consider only unsupervised clustering algorithms. In particular, we employ the Growing Neural Gas [Fritzke, 1995], the DBSCAN [Ester et al., 1996], and the unsupervised $k$-windows [Tasoulis \& Vrahatis, 2004; Vrahatis et al., 2002] clustering algorithms. Next, the aforementioned unsupervised algorithms are briefly presented.

\subsubsection{Growing Neural Gas Clustering Algorithm}

The Growing Neural Gas (GNG) clustering algorithm [Fritzke, 1995] is an incremental neural network. It can be described as a graph consisting of $k$ nodes, each of which has an associated weight vector, defining the node's position in the data space and a set of edges between the node and its neighbors. During the clustering procedure, new nodes are added to the network until a maximal number of nodes is reached. GNG starts with two nodes, randomly positioned in the data space, connected by an edge. Adaptation of weights, i.e. the nodes' positions, is performed iteratively. For each data object the closest node (winner), $s_{1}$, and the closest neighbor of the winner node, $s_{2}$, are identified. These two nodes are connected by an edge. An age variable is associated with each edge. When the edge between $s_{1}$ and $s_{2}$ is created its age is set to zero. At each learning step the age variable of all edges emanating from the winner node are increased by one. By tracing the changes of the age variable it is possible to detect inactive nodes. Edges exceeding a maximal age, $R$, and any 
$4 \quad$ N.G. Pavlidis et al.

nodes having no emanating edges are removed. The neighborhood of the winner is limited to its topological neighbors. The winner and its topological neighbors are moved in the data space toward the presented object by a constant fraction of the distance, defined separately for the winner and its topological neighbors. There is no neighborhood function, or ranking concept and thus, all topological neighbors are updated in the same manner.

\subsubsection{The DBSCAN Clustering Algorithm}

The DBSCAN clustering algorithm [Sander et al., 1998] relies on a density-based notion of clusters and is designed to discover clusters of arbitrary shape and to distinguish noise. More specifically, the algorithm relies on the idea that for each point in a cluster at least a minimum number of objects, MinPts, should be contained in a neighborhood of a given radius, Eps, around it. Thus, by iteratively scanning all the points in the dataset DBSCAN forms clusters of points that are connected through chains of Eps-neighborhoods of at least MinPts points each.

\subsubsection{Unsupervised $k$-windows}

The unsupervised $k$-windows clustering algorithm [Tasoulis \& Vrahatis, 2004; Vrahatis et al., 2002] uses a windowing technique to discover the clusters present in a dataset. More specifically, if we suppose that the dataset lies in $d$ dimensions, it initializes a number of $d$-dimensional windows over the dataset. At a next step it iteratively moves and enlarges these windows to enclose all the patterns that belong to one cluster in a window. The movement and enlargement procedures are guided by the points that lie within a window at each iteration. As soon as the movement and enlargement procedures do not alter significantly the number of points within a window they terminate. The final set of windows defines the clustering result of the algorithm. The unsupervised $k$-windows algorithm (UKW) applies the $k$-windows algorithm using a "sufficiently" large number of initial windows. The windowing technique of the $k$-windows algorithm allows for a large number of initial windows to be examined without any significant overhead in time complexity. At a final step the windows that contain a high percentage of common points from the dataset are considered to belong to the same cluster. Thus the number of clusters can be determined [Alevizos et al., 2002; Alevizos et al., 2004; Tasoulis \& Vrahatis, 2004].

\subsection{Feedforward Neural Networks}

Artificial Neural Networks (ANNs) have been widely employed in numerous fields and have shown their strengths in solving real-world problems. ANNs are parallel computational models comprised of interconnected adaptive processing units (neurons), characterized by an inherent propensity for storing experiential knowledge. They resemble the human brain in two fundamental respects; firstly, knowledge is 
acquired by the network from its environment through a learning process, and secondly, interneuron connection strengths (known as weights) are employed to store the acquired knowledge [Haykin, 1999].

Numerous neural network models have been proposed, but multilayered Feedforward Neural Networks (FNNs) are the most common. In FNNs neurons are arranged in layers and there are connections between neurons in one layer to the neurons of the following layer. The learning rule typically used for FNNs is supervised training. Two critical parameters for the successful application of FNNs are the appropriate selection of the network architecture and the training algorithm. For the general problem of function approximation, the universal approximation theorem, proved in [White, 1990] states that:

Theorem 2.1. Standard Feedforward Networks with only a single hidden layer can approximate any continuous function uniformly on any compact set and any measurable function to any desired degree of accuracy.

An immediate implication of the above theorem is that any lack of success in applications must arise from inadequate learning and/or an insufficient number of hidden units and/or the lack of a deterministic relationship between the input patterns and the desired response (target).

In the context of time series modeling the inputs to the FNN typically consist of a number of delayed observations, while the target is the next value of the series. The universal myopic mapping theorem [Sandberg \& Xu, 1997a; Sandberg \& Xu, 1997b] states that any shift-invariant map can be approximated arbitrarily well by a structure consisting of a bank of linear filters feeding an FNN. An implication of this theorem is that, in practice, FNNs alone can be insufficient to capture the dynamics of a non-stationary system [Haykin, 1999]. This is also verified by the results presented in this paper.

The selection of the optimal network architecture for a specific task remains up to date an open problem. An upper bound on the architecture of an FNN designed to approximate a continuous function defined on the unit cube in $\mathbb{R}^{n}$ is given by the following Theorem [Pinkus, 1999]:

Theorem 2.2. On the unit cube in $\mathbb{R}^{n}$ any continuous function can be uniformly approximated, to within any error by using a two hidden layer network having $2 n+1$ units in the first layer and $4 n+3$ units in the second layer.

\subsection{Supervised Training of Neural Networks}

The supervised training process is an incremental adaptation of the weights that propagate information between the neurons. Learning in FNNs is achieved by minimizing the network error using a batch, also called off-line, or a stochastic, also called on-line, training algorithm.

Batch training is considered as the classical machine learning approach. In time series applications, a set of patterns is used for modeling the system, before the 
6 N.G. Pavlidis et al.

network is actually used for prediction. In this case, the goal is to find a minimizer $w^{*}=\left(w_{1}^{*}, w_{2}^{*}, \ldots, w_{n}^{*}\right) \in \mathbb{R}^{n}$, such that:

$$
w^{*}=\min _{w \in \mathbb{R}^{n}} E(w),
$$

where $E$ is the batch error measure of the FNN, whose $l$-th layer $(l=1, \ldots, M)$ contains $N_{l}$ neurons:

$$
E=\frac{1}{2} \sum_{p=1}^{P} \sum_{j=1}^{N_{M}}\left(y_{j, p}^{M}-t_{j, p}\right)^{2}=\sum_{p=1}^{P} E_{p} .
$$

In the above relation, the error function is based on the squared difference between the actual output value at the $j$-th output layer neuron for pattern $p, y_{j, p}^{M}$, and the target output value, $t_{j, p} . E_{p}$ is the error of the $p$-th pattern and $p$ is the index over the input-output pairs. To predict the next value of the time series, there is only one output neuron $\left(N_{M}=1\right)$. On the other hand, when the problem is formulated as a classification task the value of $N_{M}$ can vary according to the number of classes.

Supervised training is a difficult task since, in general, the dimension of the weight space is very high and the function $E$ generates a complicated surface, characterized by multiple local minima and broad flat regions adjoined to narrow steep ones.

In on-line training, the FNN weights are updated after the presentation of each training pattern. On-line training may be the appropriate choice for learning a task either because of the very large (or even redundant) training set, or because of the slowly time-varying nature of the task. Although batch training seems faster for small-size training sets and networks, on-line training is probably more efficient for large training sets and FNNs. It often helps to avoid local minima and provides a more natural approach for learning non-stationary tasks, such as time series modeling and prediction. On-line methods seem to be more robust than batch methods as errors, omissions, or redundant data in the training set can be corrected, or ejected during the training phase.

In this paper we have employed and compared four algorithms for batch training and one on-line training algorithm. The batch training algorithms were the well-known Resilient Propagation (RPROP) [Riedmiller \& Braun, 1993], a Scaled Conjugate Gradient (SCG) [Møller, 1993] and two population based algorithms, namely the Differential Evolution algorithm (DE) [Storn \& Price, 1997] and the Particle Swarm Optimization (PSO) [Eberhart et al., 1996]. We also implemented the recently proposed Adaptive On-line BackPropagation training algorithm (AOBP) [Magoulas et al., 2001; Plagianakos et al., 2000]. Next, we briefly describe the AOBP, the DE, as well as, the PSO algorithms.

\subsubsection{The Online Neural Network Training Algorithm}

Despite the abundance of methods for learning from examples, there are only a few that can be used effectively for on-line learning. For example, the classic batch 
training algorithms can not straightforwardly handle non-stationary data. Even when some of them are used in on-line training the problem of "catastrophic interference" appears, in which training on new examples interferes excessively with previously learned examples, leading to saturation and slow convergence [Sutton \& Whitehead, 1993].

Methods suited to on-line learning are those that can handle time-varying data, while at the same time, require relatively little additional memory and computation in order to process one additional example. The AOBP method proposed in [Magoulas et al., 2001; Plagianakos et al., 2000] belongs to this class of methods.

The key features of this method are the low storage requirements and the inexpensive computations. At each iteration, the $d$-dimensional weight vector is evaluated using the following update formula:

$$
w^{g+1}=w^{g}-\eta^{g} \nabla E\left(w^{g}\right) .
$$

To calculate the learning rate for the next iteration, $\eta^{g+1}$, AOBP uses information from the current and the previous iteration. In detail, the new learning rate is calculated through the following relation:

$$
\eta^{g+1}=\eta^{g}+K\left\langle\nabla E\left(w^{g-1}\right), \nabla E\left(w^{g}\right)\right\rangle,
$$

where $\eta$ is the learning rate, $K$ is the meta-learning rate constant (typically $K=$ $0.5)$, and $\langle\cdot, \cdot\rangle$ stands for the usual inner product in $\mathbb{R}^{d}$. This approach stabilizes the learning rate adaptation process, and previous experiments [Magoulas et al., 2001; Plagianakos et al., 2000] have shown that it allows the method to exhibit good generalization and high convergence rate.

\subsubsection{Differential Evolution Training Algorithm}

DE [Storn \& Price, 1997] is a novel minimization method designed to handle nondifferentiable, nonlinear and multimodal objective functions, by exploiting a population of $N P$ potential solutions, that is $d$-dimensional vectors, to probe the search space. At each iteration of the algorithm, called generation, $g$, three steps, mutation, recombination and selection, are performed to obtain more accurate approximations [Plagianakos \& Vrahatis, 2002]. Initially, all weight vectors are initialized by using a random number generator. At the mutation step, for each $i=1, \ldots, N P$ a new mutant weight vector $v_{g+1}^{i}$ is generated by combining weight vectors, randomly chosen from the population, and exploiting the following variation operator:

$$
v_{g+1}^{i}=\omega_{g}^{i}+\mu\left(\omega_{g}^{\text {best }}-\omega_{g}^{i}+\omega_{g}^{r 1}-\omega_{g}^{r 2}\right),
$$

where $\omega_{g}^{r 1}$ and $\omega_{g}^{r 2}$ are randomly selected vectors, different from $\omega_{g}^{i}$, and $\omega_{g}^{\text {best }}$ is the member of the current generation that yielded the lowest error function value. Finally, the positive mutation constant $\mu$, controls the magnification of the difference between two weight vectors (typically $\mu=0.8$ ).

The resulting mutant vectors are mixed with a predetermined weight vector, called target vector. This operation is called recombination, and it gives rise to the 
$8 \quad$ N.G. Pavlidis et al.

trial vector. At the recombination step, for each component $j=1,2, \ldots, d$ of the mutant weight vector a random number $r \in[0,1]$ is generated. If $r$ is smaller than the predefined recombination constant $p$ (typically $p=0.9$ ), the $j$-th component of the mutant vector $v_{g+1}^{i}$ becomes the $j$-th component of the trial vector. Otherwise, the $j$-th component of the target vector, $\omega_{g}^{i}$, is selected as the $j$-th component of the trial vector. Finally, at the selection step, the trial weight vector obtained after the recombination step is accepted for the next generation, if and only if, it yields a reduction of the value of the error function relative to the previous weight vector; otherwise, the previous weight vector is retained.

\subsubsection{Particle Swarm Optimization Training Algorithm}

PSO is a swarm-intelligence optimization algorithm capable of minimizing nondifferentiable, nonlinear and multimodal objective functions. Each member of the swarm, called particle, moves with an adaptable velocity within the search space, and retains in its memory the best position it ever encountered. At each iteration, the best position ever attained by the swarm is communicated among the particles [Eberhart et al., 1996].

Assume a $d$-dimensional search space, $\mathcal{S} \subset \mathbb{R}^{d}$, and a swarm of $N P$ particles. Both the position and the velocity of the $i$-th particle are $d$-dimensional vectors, $x_{i} \in \mathcal{S}$ and $v_{i} \in \mathbb{R}^{d}$, respectively. The best previous position ever encountered by the $i$-th particle is denoted by $p_{i}$, while the best previous position attained by the swarm is denoted by $p_{g}$. The velocity [Clerc \& Kennedy, 2002] of the $i$-th particle at the $(g+1)$-th iteration is obtained through Eq. (3). The new position of this particle is determined by simply adding the velocity vector to the previous position vector, Eq. (4).

$$
\begin{aligned}
& v_{i}^{(g+1)}=\chi\left(v_{i}^{(g)}+c_{1} r_{1}\left(p_{i}^{(g)}-x_{i}^{(g)}\right)+c_{2} r_{2}\left(p_{g}^{(g)}-x_{i}^{(g)}\right)\right), \\
& x_{i}^{(g+1)}=x_{i}^{(g)}+v_{i}^{(g+1)},
\end{aligned}
$$

where $i=1, \ldots, N P ; c_{1}$ and $c_{2}$ are positive constants (typically $c_{1}=c_{2}=2.05$ ); $r_{1}, r_{2}$ are random numbers uniformly distributed in $[0,1]$; and $\chi$ is the constriction factor (typically $\chi=0.729$ ). In general, PSO has proved to be very efficient and effective in tackling various difficult problems [Parsopoulos \& Vrahatis, 2002].

\section{Presentation of Experimental Results}

The time series considered was that of the daily spot prices of the exchange rate of the German Mark relative to the US Dollar [Keogh \& Folias, 2002]. The time period considered extends from 10/9/1986 to 8/9/1996, covering approximately ten years. The total number of observations was 2567 . The first 2317 were used to evaluate the parameters of the predictive models, while the remaining 250 , covering approximately the final year of the dataset, were used to evaluate their performance. 
At a first step we attempted to train a single FNNs to model and forecast the time series.

The first step in the analysis and prediction of time series originating from realworld systems is the choice of an appropriate time delay, $T$, and the determination of the embedding dimension, $D$. To select $T$ an established approach is to use the value that yields the first minimum of the mutual information function [Fraser, 1989]. For the considered time series no minimum occurs for $T=1, \ldots, 20$, as illustrated in Fig. 1. In this case a time delay of one is typically selected. To determine the minimum embedding dimension for state space reconstruction we applied the method of "False Nearest Neighbors" [Hegger et al., 1999; Kennel et al., 1992]. As illustrated in Fig. 1 the proportion of false nearest neighbors as a function of $D$ drops sharply to the value of 0.006 for $D$ equal to five, which is the embedding dimension that we selected, and it becomes zero for dimensions higher than seven. With this embedding dimension the number of patterns used to evaluate the parameters of the predictive models was 2312 while the performance of the models was evaluated on the last 250 patterns.
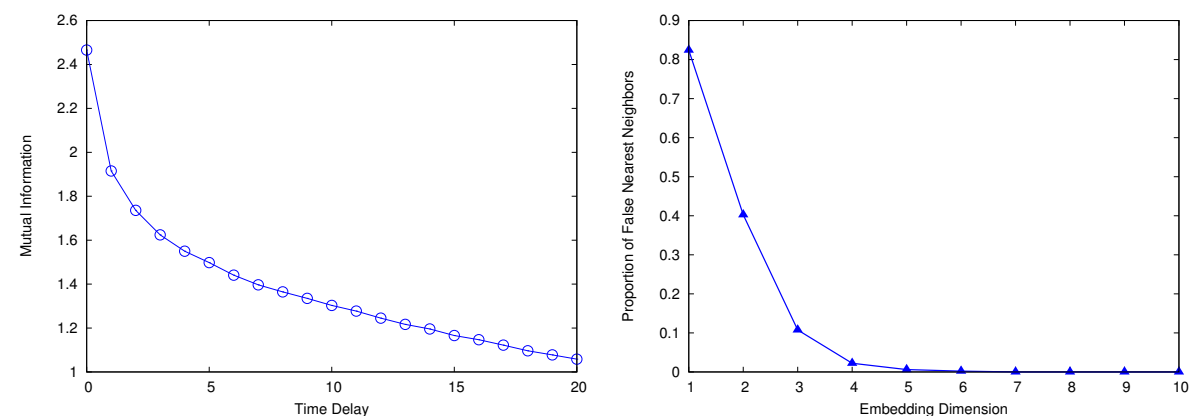

Fig. 1. Mutual information as a function of $T$ (left) and proportion of "false nearest neighbors" as a function of $D$ (right).

Having selected an embedding dimension, we tested numerous FNNs with different architectures and training algorithms, but no FNN was capable of producing a satisfactory test set prediction accuracy. In fact, the forecasts resembled a time-lagged version of the original series. Next, the three unsupervised clustering algorithms, namely GNG, DBSCAN and UKW, were applied on the patterns of training set to obtain a partition of the input space. Note that the value to be predicted (target value) by the FNNs acting as local approximators, was also included in the patterns comprising the dataset supplied to the clustering algorithms. Our experience suggests that this approach slightly improves the overall forecasting performance. Once the clusters present in the training set are identified, each pattern from the test set is assigned to one of the clusters. Since the target value for patterns in the test set is unknown the assignment is performed by not taking into consid- 
eration the additional dimension that corresponds to the target component. A test set pattern is assigned to the cluster to which the nearest (in terms of Euclidean distance) node, pattern, window center, belongs for the GNG, DBSCAN, and UKW algorithms, respectively.

We evaluate the accuracy of the FNNs by the percentage of correct sign prediction [de Bodt et al., 2001; Giles et al., 2001; Walczak, 2001]. This measure captures the percentage of forecasts in the test set for which the following inequality is satisfied:

$$
\left(\widehat{x_{t+d}}-x_{t+d-1}\right) \cdot\left(x_{t+d}-x_{t+d-1}\right)>0,
$$

where, $\widehat{x_{t+d}}$ represents the prediction generated by the FNN, $x_{t+d}$ refers to the true value of the exchange rate at period $t+d$ and, finally, $x_{t+d-1}$ stands for the value of the exchange rate at the current period, $t+d-1$. Correct sign prediction in effect captures the percentage of profitable trades enabled by the forecasting system. To successfully train FNNs capable of forecasting the direction of change of the time series, a modified, nondifferentiable, error function was implemented:

$$
E_{k}= \begin{cases}0.5 \cdot\left|x_{t+d}-\widehat{x_{t+d}}\right|, & \text { if }\left(\widehat{x_{t+d}}-x_{t+d-1}\right) \cdot\left(x_{t+d}-x_{t+d-1}\right)>0 \\ \left|x_{t+d}-\widehat{x_{t+d}}\right|, & \text { otherwise. }\end{cases}
$$

Since RPROP, SCG and AOBP are gradient based algorithms, this function is employed only when the FNNs are trained through the DE and PSO algorithms.

Numerical experiments were performed using a Clustering and a Neural Network $\mathrm{C}++$ Interface, built under the Fedora Core Linux 3.0 operating system using the GNU compiler collection (gcc) version 3.4.2. The results obtained are reported in Tables 1-3 and the accompanying figures. Each table reports the total number of clusters identified in the training set. Furthermore, it reports the number of clusters to which test set patterns were assigned. For each such cluster the number of patterns from the training set and the test set assigned to this cluster are also reported. Notice that irrespective of the clustering algorithm, a relatively small proportion of the patterns contained in the training set was actually used to generate the predictions, since training only the FNNs corresponding to the particular clusters is necessary. The accompanying figures provide candlestick plots. Each candlestick depicts for a cluster and a training algorithm the forecasting accuracy with respect to sign prediction, obtained over 100 experiments. A filled box is plotted between the first and third quartile of the data. The lines extending from each end of the box (whiskers) show the range of the data. The black line inside the box stands for the mean value of the measurements. An immediate observation from the inspection of the figures is that there are significant differences in the predictability of the different clusters, irrespective of the clustering algorithm. Moreover, within the same cluster, different training algorithms produced FNNs yielding different predictive accuracy.

For clusters 1, 3, 5 identified by the UKW algorithm and the having the corresponding FNNs trained by the DE and PSO algorithms, a mean predictive accuracy 


\begin{tabular}{ccc} 
Table 1. & UKW: results \\
\hline & Patterns in the \\
& train set & test set \\
\hline Cluster 1 & 84 & 33 \\
Cluster 2 & 82 & 57 \\
Cluster 3 & 65 & 3 \\
Cluster 4 & 239 & 67 \\
Cluster 5 & 210 & 90 \\
\hline Total number of clusters: 13. \\
Clusters used in test set: 5. \\
\hline
\end{tabular}

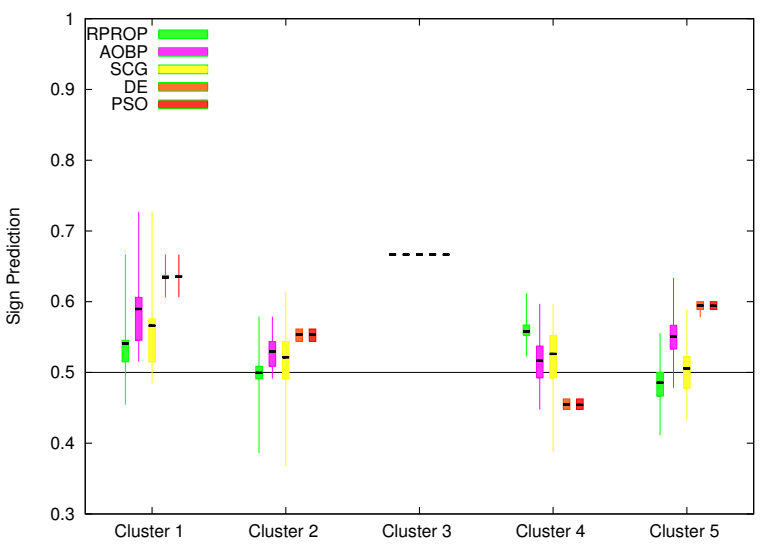

Fig. 2. Proportion of correct sign prediction based on the clustering of the input space using the UKW algorithm.

exceeding $60 \%$ was achieved. These three clusters together comprise more than $50 \%$ of the test set. However, the predictability of cluster 4 (26.8\% of the test set) is rather low. As previously mentioned, DBSCAN has the ability to identify outliers in a dataset. In this case close to $50 \%$ of the patterns of the test set were characterized as outliers. The FNN trained on these patterns produced a poor performance. On the other hand, the mean predictability for clusters 1 and 2 was around $55 \%$. Note that cluster 3 (to which four test patterns were assigned) exhibited extremely high predictability. The GNG algorithm distinguished cluster 2 for which the corresponding FNN produced a mean accuracy close to $60 \%$ irrespective of the training algorithm used. For cluster 4, PSO and DE exhibited good performance, but the other three algorithms yielded the worst performance witnessed in this study.

\section{Discussion and Concluding Remarks}

In this study, we report results from the application of unsupervised clustering algorithms, combined with feedforward neural networks in exchange rate time series forecasting. The desirable property of unsupervised clustering algorithms is that they can automatically approximate the number of partitions (clusters), thus relieving the user from making this critical, problem-specific, choice.

Combining this input space partitioning methodology with feedforward neural networks acting as local predictors for each identified cluster helps alleviate the problem of non-stationarity frequently encountered in real-life applications. Feedforward neural networks are selected as local approximation models due to their ability to cope with noise. Through this approach an improvement, compared to a global feedforward neural networks, in the one-step-ahead prediction of the direction of change of the daily spot exchange rate of the German Mark to the US Dollar was achieved. 
Table 2. DBSCAN: results

\begin{tabular}{ccc}
\hline & \multicolumn{2}{c}{ Patterns in the } \\
& train set & test set \\
\hline Outliers & 1353 & 123 \\
Cluster 1 & 95 & 59 \\
Cluster 2 & 81 & 53 \\
Cluster 3 & 4 & 4 \\
Cluster 4 & 11 & 11 \\
\hline Total number of clusters: 12. \\
Clusters used in test set: 5 .
\end{tabular}

Table 3. GNG: results

\begin{tabular}{ccc}
\hline & \multicolumn{2}{c}{ Patterns in the } \\
& train set & test set \\
\hline Cluster 1 & 90 & 57 \\
Cluster 2 & 61 & 29 \\
Cluster 3 & 94 & 6 \\
Cluster 4 & 496 & 158 \\
\hline Total number of clusters: 9. \\
Clusters used in test set: 4.
\end{tabular}

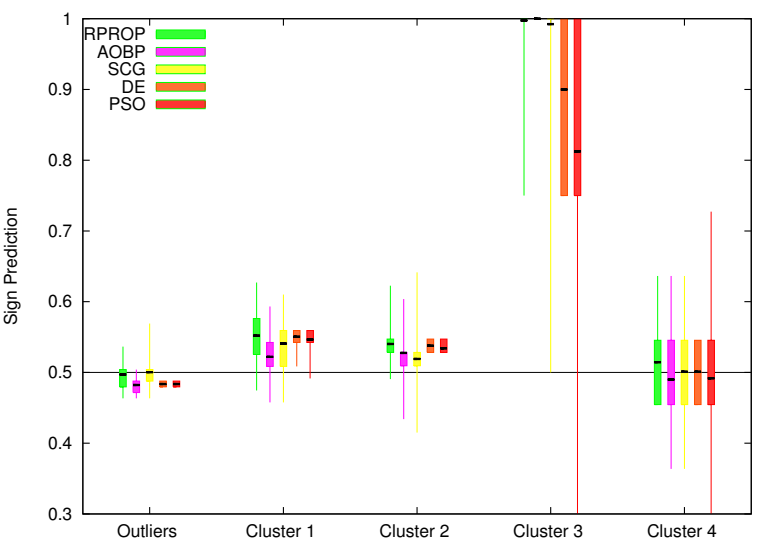

Fig. 3. Proportion of correct sign prediction based on the clustering of the input space using the DBSCAN algorithm.

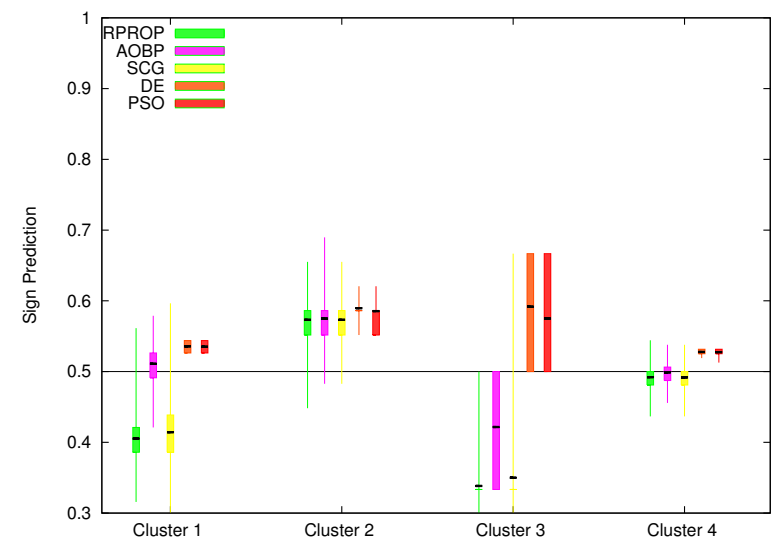

Fig. 4. Proportion of correct sign prediction based on the clustering of the input space using the GNG algorithm.

Among the unsupervised clustering algorithms considered, UKW's performance is more robust. Both the DBSCAN and the GNG algorithms however, were capable of identifying meaningful clusters that yielded increased predictability in the test set. From the training algorithms considered, FNNs trained using the AOBP training algorithm exhibited the highest maximum performance. The performance of the population based algorithms, DE and PSO, exhibited wide variations.

Future work will include the synthesis of the results of the different clustering algorithms to improve the forecasting performance in larger regions of the input space, and also the examination of other real-life time series. 


\section{Acknowledgment}

The authors thank the European Social Fund (ESF), Operational Program for Educational and Vocational Training II (EPEAEK II) and particularly the Program PYTHAGORAS, for funding this work. This work was supported in part by the "Karatheodoris" research grant awarded by the Research Committee of the University of Patras.

\section{References}

Alevizos, P., Boutsinas, B., D. K., Tasoulis \& Vrahatis, M. N. [2002] "Improving the orthogonal range search $k$-windows clustering algorithm ", Proceedings of the 14th IEEE International Conference on Tools with Artificial Intelligence, Washington, D.C., pp. 239-245

Alevizos, P., Tasoulis, D. K. \& Vrahatis, M. N. [2004] "Parallelizing the unsupervised $k$-windows clustering algorithm", Lecture Notes in Computer Science, 3019, 225-232.

Bezdek, J. C. [1981] Pattern Recognition with Fuzzy Objective Function Algorithms. (Kluwer Academic Publishers).

Cao, L. [2003] "Support vector machines experts for time series forecasting", Neurocomputing, 51, 321-329.

Clerc, M. \& Kennedy, J. [2002] "The particle swarm-explosion, stability, and convergence in a multidimensional complex space", IEEE Trans. Evol. Comput., 6(1), 58-73.

Bank of International Settlements, [2001] "Central bank survey of foreign exchange and derivative market Activity in April 2001", Bank of International Settlements.

de Bodt, E., Rynkiewicz, J. \& Cottrell, M. [2001] "Some known facts about financial data", European Symposium on Artificial Neural Networks (ESANN'2001) pp. $223-236$.

Eberhart, R. C., Simpson, P. \& Dobbins, R. [1996] Computational Intelligence PC Tools, (Academic Press).

Ester, M., Kriegel, H. P., Sander, J. \& Xu, X. [1996] "A density-based algorithm for discovering clusters in large spatial databases with noise", Proceedings of the 2nd Int. Conf. on Knowledge Discovery and Data Mining, pp. 226-231.

Fraser, A. M. [1989] "Information and entropy in strange attractors", IEEE Transactions on Information Theory, 35, 245-262.

Fritzke, B. [1995] "A growing neural gas network learns topologies", eds. Tesauro, G., Touretzky, D. S. \& Leen, T. K. (eds), Advances in Neural Information Processing Systems, (MIT Press, Cambridge MA), pp. 625-632.

Giles, L. C., , Lawrence, S. \& Tsoi, A. H. [2001] "Noisy time series prediction using a recurrent neural network and grammatical inference", Machine Learning, 44(1/2), 161-183. 
Hartigan, J. A. \& Wong, M. A. [1979] "A $k$-means clustering algorithm", Applied Statistics, 28, 100-108.

Hastie, T., Tibshirani, R. \& Friedman, J. [2001] The Elements of Statistical Learning, (Springer-Verlag).

Haykin, S. [1999] Neural Networks: A Comprehensive Foundation, (New York: Macmillan College Publishing Company).

Hegger, R., Kantz, H. \& Schreiber, T. [1999] "Practical implementation of nonlinear time series methods: The TISEAN package", Chaos, 9(2), 413-435.

Kennel, M. B., Brown, R. \& Abarbanel, H. D. [1992] "Determining embedding dimension for phase-space reconstruction using a geometrical construction", Physical Review A, 45(6), 3403-3411.

Keogh, E. \& Folias, T. [2002] The UCR Time Series Data Mining Archive.

Kohonen, T. [1997] . Self-Organized Maps, (Berlin, Springer).

Magoulas, G. D., Plagianakos, V. P. \& Vrahatis, M. N. [2001] "Adaptive stepsize algorithms for on-line training of neural networks", Nonlinear Analysis, T.M.A., 47(5), 3425-3430.

Møller, M. [1993] "A scaled conjugate gradient algorithm for fast supervised learning", Neural Networks, 6, 525-533.

Parsopoulos, K. E. \& Vrahatis, M. N. [2002] "Recent approaches to global optimization problems through particle swarm optimization", Natural Computing, $\mathbf{1}(2-3), 235-306$.

Pavlidis, N. G., Tasoulis, D. K. \& Vrahatis, M. N. [2003] "Financial forecasting through unsupervised Clustering and evolutionary trained neural networks", Proceedings of the Congress on Evolutionary Computation (CEC 2003), pp. 2314-2321.

Pavlidis, N. G., Tasoulis, D. K. \& Vrahatis, M. N. [2005] "Time series forecasting methodology for multiple-step-ahead prediction", The IASTED International Conference on Computational Intelligence (CI 2005), pp. 456-461.

Pinkus, A. [1999] "Approximation theory of the mlp model in neural networks", Acta Numerica, 143-195.

Plagianakos, V. P., Magoulas, G. D. \& Vrahatis, M. N. [2000] "Global learning rate adaptation in on-line neural network training", Proceedings of the Second International ICSC Symposium on Neural Computation (NC'2000).

Plagianakos, V. P. \& Vrahatis, M. N. [2002] "Parallel evolutionary training algorithms for 'hardware-friendly' neural networks", Natural Computing, 1, 307322 .

Principe, J. C., Wang, L. \& Motter, M. A. [1998] "Local dynamic modeling with self-organizing maps and applications to nonlinear system identification and control", Proceedings of the IEEE, 86(11), 2240-2258.

Milidiu, R. L., Machado, R. J. \& Renteria, R. P. [1999] "Time-series forecasting through wavelets transformation and a mixture of expert models", Neurocomputing, 28, 145-156. 
Riedmiller, M. \& Braun, H. [1993] "A direct adaptive method for faster backpropagation learning: the RPROP algorithm", Proceedings of the IEEE International Conference on Neural Networks. (San Francisco, CA), pp. 586-591.

Sandberg, I. W. \& Xu, L. [1997]a "Uniform approximation and gamma networks", Neural Networks, 10(5), 781-784.

Sandberg, I. W. \& Xu, L. [1997]b "Uniform approximation of multidimensional myopic maps", IEEE Transactions on Circuits and Systems I: Fundamental Theory and Applications, 44(6), 477-485.

Sander, J., Ester, M., Kriegel, H.-P. \& Xu, X. [1998] "Density-based clustering in spatial databases: the algorithm GDBSCAN and its applications", Data Mining and Knowledge Discovery, 2(2), 169-194.

Sfetsos, A. \& Siriopoulos, C. [2004] "Time series forecasting with a hybrid clustering scheme and pattern recognition", IEEE Transactions on Systems, Man, and Cybernetics Part A: Systems and Humans, 34(3), 399-405.

Storn, R. \& Price, K. [1997] "Differential evolution - A simple and efficient adaptive scheme for global optimization over continuous spaces", Journal of Global Optimization, 11, 341-359.

Sutton, R. S. \& Whitehead, S. D. [1993] "Online learning with random representations", Proceedings of the Tenth International Conference on Machine Learning, (Morgan Kaufmann), pp. 314-321.

Tasoulis, D. K. \& Vrahatis, M. N. [2004] "Unsupervised distributed clustering", IASTED International Conference on Parallel and Distributed Computing and Networks. (Innsbruck, Austria), pp. 347-351.

Vrahatis, M. N., Boutsinas, B., Alevizos, P. \& Pavlides, G. [2002] "The new $k$ windows algorithm for improving the $k$-means Clustering Algorithm", Journal of Complexity, 18, 375-391.

Walczak, S. [2001] "An empirical analysis of data requirements for financial forecasting with neural networks", Journal of Management Information Systems, 17(4), 203-222.

Weigend, A. S., Mangeas, M. \& Srivastava, A. N. [1995] "Nonlinear gated experts for time series: discovering regimes and avoiding overfitting", International Journal of Neural Systems, 6, 373-399.

White, H. [1990] "Connectionist nonparametric regression: Multilayer feedforward networks can learn arbitrary mappings", Neural Networks, 3, 535-549.

Yao, J. \& Tan, C. L. [2000] "A case study on using neural networks to perform technical forecasting of forex", Neurocomputing, 34, 79-98. 